\title{
Native or promiscuous? Analyzing putative dimethylsulfoniopropionate lyases using a substrate proofing approach
}

Lei Lei, Kesava Phaneendra Cherukuri, Diana Meltzer, Uria Alcolombri \& Dan S. Tawfik

Department of Biomolecular Sciences, Weizmann Institute of Science, Rehovot 76100, Israel.

* Correspondence to: dan.tawfik@weizmann.ac.il

\begin{abstract}
Enzyme promiscuity is widely spread. Foremost, within superfamilies, the native function of one enzyme is typically observed as promiscuous activity in related enzymes. The native function usually exhibits high catalytic efficiency while promiscuous activities are weak, but this is not always the case. Thus, for certain enzymes it remains questionable whether their currently known activity is native or promiscuous. Dimethylsulfon-iopropionate (DMSP) is an abundant marine metabolite cleaved via $\beta$ elimination to release dimethylsulfide (DMS). Eight different gene families have been identified as putative DMSP lyases, 5 of them belonging to the same superfamily (cupinDLL; see the accompanying paper). Some of these enzymes exhibit very low activity, but this can be due to suboptimal folding or reaction conditions. We developed a substrate profiling approach with the aim of distinguishing native DMSP lyases from enzymes that promiscuously act as DMSP lyases. In a native DMSP lyase, relatively small changes in the structure of DMSP should induce significant activity drops. We thus profiled substrate selectivity by systematically modifying DMSP while retaining reactivity. Three enzymes that exhibit the highest activity with DMSP also exhibited high sensitivity to perturbation of DMSP's structure (Alma, DddY, and DddL). The two enzymes with the weakest DMSP lyase activity also showed the highest crossreactivity (DddQ, DddP). Combined with other indications, it appears that the DMSP lyase activity of DddQ and DddP is promiscuous although their native function remains unknown. Systematic
\end{abstract}


substrate profiling could help identify and assign potential DMSP lyases, and possibly applied to other enzymes.

Key words: Enzyme promiscuity, enzyme superfamily, substrate profiling, dimethylsulfide.

Abbreviations: DMSP, dimethylsulfoniopropionate; DMS, dimethylsulfide; cupin-DLL, cupin DMSP lyase and lyase-like.

Funding: Financial support by the Estate of Mark Scher, and the Sasson \& Marjorie Peress Philanthropic Fund, are gratefully acknowledged. D.S.T. is the Nella and Leon Benoziyo Professor of Biochemistry. 


\section{Introduction}

Enzymes are renowned for being able to convert one specific substrate into a given product with high turnover rates. Nonetheless, enzyme promiscuity, i.e., the ability to transform substrates other than the native substrate, or even to catalyze a different reaction, is widely spread. ${ }^{1-3}$ Take nitrogenase, to name just one example. Its native, physiological function is to reduce $\mathrm{N}_{2}$ to $\mathrm{NH}_{3}$. However, this enzyme reduces alternative substrates such as cyanide or acetylene, ${ }^{4,5}$ and the latter is widely applied as a surrogate of nitrogenase activity. ${ }^{6}$ Promiscuity is also a major driving force in the natural evolution of new enzyme functions. $1,2,7$

Within enzyme superfamilies, promiscuity reflects the catalytic mechanism shared by all superfamily members. A common key chemical step enables a range of reactions that can be catalyzed by the same configuration of catalytic active-site residues. For example, the enolase superfamily's key chemical step is proton abstraction from a carbon adjacent to a carboxylate. This step underlies different reactions including dehydration, isomerisation or lactonisation, with a wide range of different substrates. ${ }^{8}$ Owing to the shared chemistry, the native function of one superfamily member is often manifested as a promiscuous activity in related members and vice versa. This phenomenon helps unravel the evolutionary origins of enzymes, ${ }^{9}$ but has also led to mis-annotations. ${ }^{10}$ Take for example an enzyme called serum paraoxonase/ arylesterase, or PON1. This enzyme was characterized with synthetic substrates including paraoxon and phenyl acetate that are hydrolyzed with relatively high rates $\left(k_{c a t} / K_{M} \sim 10^{4-} 10^{6} \mathrm{M}^{-1} \mathrm{~s}^{-}\right.$ $\left.{ }^{1}\right)$. However, PON1 turned out the be a lactonase, as indicated by systematically testing of different substrates, of which, only lactones exhibited a selectivity profile that is in agreement with a native function. ${ }^{11}$ Overall, although the native function typically occurs with high catalytic efficiency while promiscuous activities are weak, this criterion is not universally valid. In fact, the kinetic parameters for both native functions and promiscuous activities are distributed over a wide range. ${ }^{12}$ Thus, determining whether the known activity of an enzyme is native or promiscuous can be a challenge. 
We encountered this challenge in the case of DMSP lyases. Many different genes encoding enzymes that catalyze the $\beta$-elimination of DMSP to release DMS a compound known as the 'smell of the sea', and acrylate are known (Scheme).

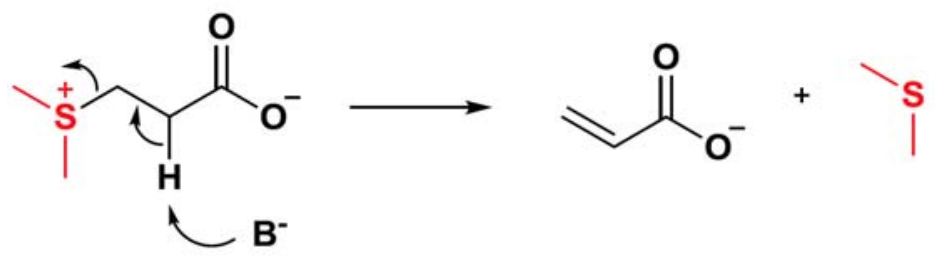

These putative DMSP lyases come from different families. Seven bacterial families, dubbed Ddd+, are known: DddD, a DMSP CoA-transferase-lyase (that generates 3hydroxypropionyl-CoA rather than acrylate), ${ }^{13,14}$ DddP that belongs to the M24 proteinase family; ${ }^{15}$ and DddQ, ${ }^{16} \mathrm{DddL}^{17}{ }^{17} \mathrm{DddW}^{18}{ }^{18} \mathrm{DddK}^{19}$ and $\mathrm{DddY}^{20,21}$ that all belong to a superfamily dubbed cupin-DLL as described in the accompany paper. One eukaryote family has also been discovered, Alma DMSP lyases, that belong to yet another superfamily (Asp/Glu racemase). ${ }^{14}$ Representatives from these families all show DMSP lyase activity, albeit at very different levels - the $k_{c a t} / K_{M}$ values range from $<1 \mathrm{M}^{-1} \mathrm{~s}^{-1} 16$, 22-24 up to $10^{6} \mathrm{M}^{-1} \mathrm{~s}^{-1} \cdot{ }^{20,25}$ However, in itself, the low activity of some DMSP lyases such as DddQ and DddP is insufficient to indicate that DMSP lysis is merely a promiscuous activity of these enzymes. Low activity can also be the outcome of other factors, including mis-folding due to heterogeneous expression, incorrect cofactors such as metal ions, and/or suboptimal reaction conditions.

To distinguish enzymes whose native function is DMSP lyase from those in which this activity is likely to be promiscuous, we have developed an approach of substrate profiling. We have examined a series of DMSP analogues in which DMSP's structure was systematically perturbed while retaining reactivity. We observed systematic behavior indicating high sensitivity to perturbation in some enzymes versus high cross-reactivity in others. Further, the specific activity of the 7 enzymes tested was found to be anticorrelated with their cross-reactivity. Overall, our results indicate that while DddL, DddY and Alma are bona fide DMSP lyases, the activity of DddQ and DddP is merely promiscuous. 


\section{Results}

\section{Substrate profiling of DMSP lyases}

The rationale underlying substrate profiling is that, principle, if a given substrate is the enzyme's native substrate, the enzyme's active-site would have largely been shaped to fit this particular substrate. Thus, relatively small changes in the substrate's structure are expected to induce relatively large drops in activity. True, the essence of enzyme promiscuity is acceptance of alternative substrates, sometimes with little resemblance to the original substrate and also in a selective manner. ${ }^{26}$ Nonetheless, selectivity is observed in most enzymes including promiscuous ones, and especially in enzymes that evolved for one particular substrate (in contrast to broad-specificity enzymes). As rules of thumb, substrates smaller than the original one will be accepted more readily than bigger ones (due to steric clashes) and increasingly larger substrates will show increasingly lower activity, and, perturbations next to where the key chemical step occurs would have larger impact.

In view of the above, a series of DMSP analogues was synthesized in which the potential for $\beta$-elimination and release of a dialkylsulfide product was retained (Figure 1A). The modifications included increasingly large dialkylsulfonyl leaving groups (EMSP, DESP, cDESP) and addition of methyl groups to the propionate moiety, either on the $\alpha$ carbon from which a proton is abstracted (2-methyl-DMSP) or on the adjacent methylene (3-methyl-DMSP). None of these analogues matches a known natural metabolite except for DMSP of course.

We tested representatives of 6 bacterial Ddd+ families (all bacterial families except DddD which is a CoA-ligase/DMAP lyase confirmed as a bona fide DMSP lyase, $\operatorname{ref}^{14}$ ) and also the algal E. huxeleyi Alma ${ }^{27}$. The bacterial DddY was expressed, purified and characterized as described in the accompanying paper. DddL could not be purified, but could be assayed in crude E. coli cell lysates (see also accompanying paper). DddK, DddW and DddP were cloned, expressed as purified as described in the Methods section. Specific activities and kinetic parameters are given in Table 1. These roughly match the 
reported ones although considerable differences were observed. For example, our preparation of Ruegeria pomeroyi DSS-3 DddQ exhibited >100-fold higher specific activity compared to a previous report. ${ }^{16,28}$ For DddK and DddW, although $K_{M}$ values have been reported, ${ }^{29,30}$ we observed no saturation with DMSP concentrations up to 10 $\mathrm{mM}$ and could therefore only measure $k_{c a t} / K_{M}$ (Supplemental Figure S1). Since all these bacterial enzymes are metal-dependent, activity differences may stem from different preparations containing different metals (but also from different assay conditions, e.g. buffer capacity, see Methods). Metal composition may have a different effect on the native compared to promiscuous activities, but these differences primarily regard catalytic promiscuity, i.e., different reactions. ${ }^{31}$ Foremost, our substrate profiling approach regards the measurement of relative activities of DMSP analogues compared to DMSP, and hence the results should be relevant regardless of differences in specific activities. Indeed, the specific activities of the 7 tested enzymes with DMSP vary by $>3,000$-fold (Table 1). Thus, the enzymes were applied at different concentrations in accordance with their specific activities. The initial rates observed with each substrate analogue, for release DMS, or of the corresponding dialkyl sulfide, were compared to the initial rate of the same enzyme with DMSP (Figure 1B).

\section{Alma1, DddL and DddY exhibit the expected DMSP-tailored selectivity}

As can be seen in Figure 1B, the enzymes tested can be readily divided to two groups: highly selective enzymes (Alma1, DddY and DddL) and less, or evidently non-selective enzymes (DddK, DddW, DddQ, and DddP respectively). Specifically, Alma and DddL failed to accept any of the applied DMSP analogues under the tested conditions (enzyme concentrations and initial rates for DMS release with DMSP are noted in the legend). DddY showed some cross-reactivity. However, a significant and systematic decrease in activity was observed with increased size of substituents on the dialkylsulfide leaving group. Foremost, almost no activity was observed with analogues contacting an extra methyl group close to where proton abstraction occurs (2-methylDMSP and 3-methyl-DMSP). 
Overall, profiling with DMSP supports the hypothesis that Alma1, DddY and DddL are bona fide DMSP lyases. This conclusion is also supported by their relatively high specific activity (elaborated below). However, in the other 4 bacterial Ddd+ families, low selectivity toward DMSP is evident.

\section{DddQ and DddP are highly likely to be promiscuous DMSP lyases}

DddQ exhibited a trend that is in clear contrast to the selectivity observed in Alma1, DddY and DddL. Modifications of the leaving group, including the bulkiest one (CDESP) hardly affected the rate. Foremost, DddQ catalyzes the $\beta$-elimination of 3-methyl-DMSP nearly 20 times faster compared to DMSP. Considering that 3-methyl-DMSP is not a natural metabolite (as much as is known), DddQ is highly likely to be an enzyme that catalyzes a reaction other than DMSP lysis.

That DddQ is highly likely to be an enzyme whose native activity is not DMSP lyase is also supported by the genome neighborhood analysis presented in the accompanying paper. In bacterial genomes, enzymes that are functionally related are typically found in gene clusters, or even in the same operon. DddQ's genome neighborhood does not seem to include acrylate catabolizing enzymes while genes encoding such enzymes are observed in DddD and DddY (see accompanying paper). In fact, the genome neighborhood suggests that DddQ is part of pathways(s) that catabolize proline-betaine or/and hydroxyproline-betaine. That 3-methyl-DMSP is a much better substrate for DddQ also suggests that DddQ's real substrate is bulkier than DMSP.

Given the pattern of cross-reactivity, and its low activity, DddP is also likely to promiscuously act as DMSP lyase. The genome context of DddP is highly conserved (Supplemental figure S2). However, acrylate catalbolizing genes are not observed in DddP's vicinity. The proximity of murein transglycosylase, type I glutamate-ammonia ligase, and a nitrogen regulatory protein, suggest that this gene cluster is involved in peptidoglycan recycling, cell division or nitrogen metabolism. However, what is the primary enzymatic function of $D d d P$, or of $D d d Q$, remains unknown. 


\section{Discussion}

Substrate specificity is never absolute, and many, if not most enzymes accept alternative substrates, promiscuously, or because they a priori evolved with broad substrate specificity. ${ }^{1}$ Identifying whether a given activity is promiscuous or native can be non-trivial for certain enzymes, and especially for enzymes in specialized metabolism. This challenge further intensifies in cases where specific activity is low, because low activity can also be the outcome of suboptimal expression or/and reaction conditions. In the case of DMSP lyases, proteins from 4 different superfamilies are known whose DMSP lyase activity spans from 0.4 up to 1,300 Units (Table 1). Nonetheless, there are clear indications for suboptimal folding, and also issues related to the incorporation of the catalytic metal in some of these enzymes; DddL, for example, could not be purified and could only be assayed in crude lyases (see accompanying paper). To circumvent these hurdles, we developed a substrate profiling approach.

Quantifying substrate selectivity. Having obtained the substrate selectivity profiles of 7 different enzymes, we asked how correlated is their selectivity toward DMSP with their the level of their DMSP lyase activity. To examine such a correlation, a quantitative measure of enzyme selectivity had to be developed. Previous works provided quantitative measures of promiscuity, but these measures were designed to assess how broad is the substrate acceptance of a given enzyme. In contrast, we aimed at examining how likely is DMSP to be the native substrate. The more selective an enzyme is toward DMSP, the lower is the average relative activity with various DMSP analogues. In the simplest manner, a cross-reactivity index, $I_{C R}$, can be defined as:

[Eq. 1] $\quad I_{C R}=\frac{\sum_{i=1}^{N} e_{i}}{N}$

; whereby $N$ is the number of tested DMSP analogues, and $e_{i}$ is the activity relative to DMSP per each DMSP analogue. If an enzyme cleaves only DMSP, $I_{C R}=0$, as is the case with Alma1 (Table 2). In principle, $I_{C R}$ is expected to be in the range of 0 up to 1 . 
However, for DddQ the $I_{C R}$ value is $>>1$ due to the activity with one analogue being 19fold higher than with DMSP.

The above-described index has two drawbacks. Firstly, substrate analogues taken with rates much higher than the reference substrate shift certain enzymes drastically off scale (e.g. DddQ). Secondly, all analogues have the same weight. However, the applied substrate analogues structurally deviate from DMSP to different degrees - for example, cDESP presents a more dramatic structural perturbation than EMSP, and hence lack of cross-reactivity with the former is more indicative of a pocket shaped for DMSP. In principle, the more variable is the activity of different enzymes with a given analogue, the more relevant this analogue is as a reporter of the active-site's complementarity to DMSP (in analogy to entropy, $H$, values applied in Ref. ${ }^{32}$ ). To address the first issue, the activity was normalized per analogue (i.e., dividing each activity $\left(e_{i}\right)$ by the highest activity observed with this analogue, $\left.e_{i}^{\max }\right)$. To address the second issue, for each analogue, the coefficient of variation, $C_{v}{ }^{i}$, was calculated (the standard deviation for the normalized activities for all tested enzymes divided by the corresponding mean). The $C_{v}{ }^{i}$ was applied to give each analogue a different weight. As can be seen in Table 1 , the $C_{v}$ values span within a reasonable range $(0.7-2.4)$ and systematically reflect the structural deviations (gradually increasing from 0.7 to 1.14 with the bulkiness of the leaving group, and being with the highest for modifications close to the abstracted proton). The weighted cross-reactivity index ${ }^{*} I_{C R}$, is thus given by: [Eq. 2]

$$
{ }^{*} I_{C R}=\sum_{i=1}^{N}\left(\frac{e_{i}}{e_{i}^{\text {max }}}\right) C_{v}^{i}
$$

Activity and selectivity correlate. Both cross-reactivity indexes seem to correlate with specific activity: the most active DMSP lyases are also the ones showing the clearest signature of a DMSP tailored active-site, and vice versa (Figure 2). The correlation is, as expected, not perfect. Some of the deviations may relate to suboptimal expression or/and reaction conditions; foremost, given the failure to purify DddL, this enzyme's specific activity is most probably underestimated (see accompanying paper). Nonetheless, DddP and DddQ clearly stand out by both criteria - i.e., they exhibit the 
lowest DMSP lyase activity as well as the lowest selectivity toward DMSP. This conclusion is in agreement with the genome context analysis (Supplemental figure S2; for DddQ see accompanying paper).

Overall, our analysis suggests that the DMSP lyases reported to date represent an entire range of evolutionary states. Some enzymes are fully diverged DMSP lyase specialists such as Alma, DddY and DddL. Other enzymes, most probably DddK and DddW, may comprise bi-functional intermediates - namely enzymes that possess the ancestral function (whatever this function might be) and have also evolved to have DMSP lyase activity. Bi-functional enzymes are common, especially in specialized metabolism. ${ }^{1,33-36}$ In other enzymes, DddQ and DddP in most likelihood, the DMSP lyase may be merely promiscuous, i.e., a coincidental activity with no physiological relevance. Substrate profiling seems to reliably report these evolutionary states. Substrate profiling may be also valuable for mapping DMSP lyase activities of unknown origins (i.e., by examining if they correspond to a highly selective DMSP lyase, or alternatively match the profile of one of the known promiscuous enzymes). Systematic substrate profiling might also be applicable to other enzyme classes in which it remains unclear whether the presently known activity in native or promiscuous.

\section{Material and Methods}

Enzyme cloning, expression and purification. The genes $d d d K$ and $d d d W$ were synthesized by Gen9. The synthesized gene fragments $d d d K$ and $d d d W$ were amplified and the PCR product was digested with Ncol and HindIII, and then cloned into the expression vector pET28a. This allowed DddK and DddW to be expressed with a Cterminal His-tag. Expression plasmid pET21a encoding Roseovarius nubinhibens dddP, and Ruegeria pomeroyi DSS-3 dddQ, were kindly provided by Professor Andrew Johnston, University of East Anglia. The recombinant enzymes were expressed in E. Coli BL21 (DE3). Cells were grown for overnight in $5 \mathrm{~mL} \mathrm{LB}$ medium at $37^{\circ} \mathrm{C}$. These cultures (1 $\mathrm{mL}$ ) were used to inoculate 1 liter LB cultures that were subsequently grown at $37^{\circ} \mathrm{C}$ to $\mathrm{OD}_{600 \mathrm{~nm}}$ of $0.6-0.8$. The growth temperature was reduced to $16^{\circ} \mathrm{C}$, and enzyme 
expression was induced with $0.1 \mathrm{mM}$ IPTG. Following overnight growth at $16^{\circ} \mathrm{C}$, the cells were harvested by centrifugation at $4^{\circ} \mathrm{C}$. Cells were re-suspended in $50 \mathrm{~mL}$ lysis buffer (100mM Tris- $\mathrm{HCl}$ pH 8.0, $100 \mathrm{mM} \mathrm{NaCl}, 1 \mathrm{mM} \mathrm{CaCl}_{2}, 10 \mathrm{mg}$ lysozyme and $10 \mu \mathrm{g}$ bezonase and lysed by sonication. Following centrifugation at $8,000 \times \mathrm{g}$ for 20 mins, the clarified cell lysates were loaded on $2 \mathrm{~mL}$ Ni-NTA agarose beads (Millipore). Binding was performed at $4^{\circ} \mathrm{C}$, and beads were washed with $50 \mathrm{~mL}$ lysis buffer followed by $100 \mathrm{~mL}$ lysis buffer with $35 \mathrm{mM}$ imidazole. The enzymes were eluted with $150 \mathrm{mM}$ imidazole. Fractions were analyzed by SDS-PAGE and assayed for DMSP lyase activity, combined, and the purified enzyme was concentrated by ultrafiltration (Amicon). Final enzyme concentrations were determined by the BCA assay. DddY, and DddL cloning, expression, and expression were described in accompanying paper, and Alma1 was expressed as described. ${ }^{27,37}$ To obtain the kinetic parameters, recombinant DddK, DddW and DddQ enzymes (at 8, 15 and $200 \mu \mathrm{g} / \mathrm{mL}$ respectively were reacted with DMSP at different concentrations up to $10 \mathrm{mM}$ for 5 mins (at these enzyme concentrations, rates of release were found to be linear up to $5 \mathrm{~min}$ ). The amount of, the released DMS was measured to derive the initial rates (Supplemental Figure S1).

DMSP lyase specific and cross activity assays. DMS release was measured as previously described. ${ }^{10}$ Briefly, freshly prepared $100 \mathrm{mM}$ Tris- $\mathrm{HCl}$ pH 8.0 with $100 \mathrm{mM} \mathrm{NaCl}$ was used for the enzymatic assays supplemented with $10 \mathrm{mM}$ DMSP as default. High buffer capacity is critical for assaying this reaction. Firstly, DMSP and its analogues are applied as hydrochloride salt as may just shift the $\mathrm{pH}$. Additionally, every molecule of DMSP, or DMSP analogue, cleaved also releases one proton that affects $\mathrm{pH}$. Reactions were performed at $30{ }^{\circ} \mathrm{C}$ (5 min, typically) and terminated by 1000 -fold dilution into $30 \mathrm{~mL}$ chilled $10 \mathrm{mM}$ glycine pH 3.0 in sealed glass vials. Enzyme concentration were typically as follows: Ehux-Alma1, 0.3ug/mL; Sym-Alma1, $1 \mu \mathrm{g} / \mathrm{mL}$; Desufovibrio DddY 20ng/mL; Alcaligenes DddY 50ng/mL; DddW, $15 \mu \mathrm{g} / \mathrm{mL}$; DddQ, $100 \mu \mathrm{g} / \mathrm{mL} ;$ DddK, $8 \mu \mathrm{g} / \mathrm{mL}$. DddL was assayed in crude lysate, at an estimated concentration of $8 \mathrm{ug} / \mathrm{mL}$. DMS levels were determined using an Eclipse 4660 Purge-and-Trap Sample Concentrator system (OI Analytical) followed by separation and detection using GC-FPD (HP 5890) equipped with 
RT-XL sulfur column (Restek). All measurements were calibrated using DMS standards. Activity measurements of DMSP analogues were calibrated using the corresponding dialkylsulfides (ethyl methyl sulfite for EMSP, diethyl sulfite for DESP, and tetrahydrothiopene for CDESP).

DMSP analogues synthesis. In general, all chemicals were purchased from SigmaAldrich and other commercial suppliers in reagent grade and used without further purification. Solvents are of AR grade and used without further purification. Deuterated solvents were from Sigma and Cambridge Isotope Laboratories, Inc. ${ }^{1} \mathrm{H}$ and ${ }^{13} \mathrm{C} N M R$ were recorded on BRUKER AVANCE III-400 (400 MHz) in $\mathrm{D}_{2} \mathrm{O}$ or $\mathrm{CD}_{3} \mathrm{OD}$ and all the signal positions were recorded in $\delta \mathrm{ppm}$ with abbreviations $\mathrm{s}, \mathrm{d}, \mathrm{t}, \mathrm{q}, \mathrm{m}$ and dd denoting singlet, doublet, triplet, quartet, multiplet and doublet of doublet respectively. All NMR chemical shifts were referenced to residual solvent peaks $\left(C_{3} \mathrm{OD} \delta=3.31 \mathrm{ppm}\right.$ and $\mathrm{D}_{2} \mathrm{O}$ $\delta=4.79 \mathrm{ppm}$ ). Coupling constants $J$, were registered in $\mathrm{Hz}$. HRMS was determined with Xevo G2-XS QTOF mass spectrometer by electrospray ionization. The purity of all the compounds was more than $90 \%$ by ${ }^{1} \mathrm{H}$ NMR.

The various DMSP analogues were synthesized by Michael addition of the corresponding dialkylsulfide and acrylic acid, or acrylic acid derivative, essentially as described. ${ }^{37}$ Typically, the corresponding acrylic acid (1 equivalent) was dissolved in $2 \mathrm{M}$ aqueous $\mathrm{HCl}$ ( $10 \mathrm{~mL}$ for $1 \mathrm{gr}$ ), and the respective dialkylsulfide (3-6 molar equivalents) was added portion-wise. The reaction mixture was refluxed at $80^{\circ} \mathrm{C}$ for $3-12$ hours. After cooling to room temperature, the solvent and the excess of unreacted dialkylsulfide were removed under reduced pressure. In the case of EMSP, DESP, CDESP, the resulting crude product (hydrochloride salt; solid, or syrup) was purified by recrystallization from isopropanol and in the case of 2-methyl DMSP and 3-methyl DMSP, the crude product hydrochloride salt; solid, or syrup) was triturated with diethyl ether. The products were dried under vacuum to obtain the hydrochloride salts. The products identity and purity were determined by ${ }^{1} \mathrm{H}-\mathrm{NMR}(400 \mathrm{MHz}),{ }^{13} \mathrm{C}-\mathrm{NMR}(101 \mathrm{MHz})$ and HRMS analysis (NMR spectra are provided in Supplemental Figure S3).

DESP: obtained from diethyl sulfide and acrylic acid in $42 \%$ yield as a white solid. 
${ }^{1} \mathrm{H}$ NMR $\left(400 \mathrm{MHz}, \mathrm{D}_{2} \mathrm{O}\right) \delta 3.51(\mathrm{t}, J=6.9 \mathrm{~Hz}, 2 \mathrm{H}), 3.38(\mathrm{q}, J=7.4 \mathrm{~Hz}, 4 \mathrm{H}), 3.00(\mathrm{t}, J=6.8$ $\mathrm{Hz}, 2 \mathrm{H}), 1.47$ (t, $J=7.4 \mathrm{~Hz}, 6 \mathrm{H}) ;{ }^{13} \mathrm{C}$ NMR $\left(101 \mathrm{MHz}, \mathrm{D}_{2} \mathrm{O}\right) \delta 173.87,33.48,28.88,7.89$. HRMS $m / z$ calcd for $\mathrm{C}_{7} \mathrm{H}_{15} \mathrm{O}_{2} \mathrm{~S}^{+}$is 163.0793 , found 163.0801 .

EMSP: obtained from ethyl-methyl sulfide and acrylic acid in $44 \%$ yield as a colorless oil.

${ }^{1} \mathrm{H}$ NMR $\left(400 \mathrm{MHz}, \mathrm{D}_{2} \mathrm{O}\right) \delta 3.62-3.55(\mathrm{~m}, 1 \mathrm{H}), 3.52$ - $3.45(\mathrm{~m}, 1 \mathrm{H}), 3.45$ - $3.38(\mathrm{~m}, 1 \mathrm{H})$, $3.38-3.29(\mathrm{~m}, 1 \mathrm{H}), 3.01(\mathrm{t}, J=6.8 \mathrm{~Hz}, 2 \mathrm{H}), 2.92(\mathrm{~s}, 3 \mathrm{H}), 1.47(\mathrm{t}, J=7.4 \mathrm{~Hz}, 3 \mathrm{H}) ;{ }^{13} \mathrm{C} N M R$ $\left(101 \mathrm{MHz}, \mathrm{D}_{2} \mathrm{O}\right) \delta 173.83,36.49,36.29,28.66,21.90,7.79$. HRMS $\mathrm{m} / \mathrm{z}$ calcd for $\mathrm{C}_{6} \mathrm{H}_{13} \mathrm{O}_{2} \mathrm{~S}^{+}$is 149.0636 , found 149.0641 .

CDESP: obtained from tetrahydrothiophene and acrylic acid in $20 \%$ yield as a white solid.

${ }^{1} \mathrm{H} N M R\left(400 \mathrm{MHz}, \mathrm{D}_{2} \mathrm{O}\right) \delta 3.69-3.57(\mathrm{~m}, 2 \mathrm{H}), 3.55-3.47(\mathrm{~m}, 2 \mathrm{H}), 3.44(\mathrm{t}, J=6.8 \mathrm{~Hz}, 2 \mathrm{H})$, $3.00(\mathrm{t}, J=6.8 \mathrm{~Hz}, 2 \mathrm{H}), 2.44-2.24(\mathrm{~m}, 4 \mathrm{H}) ;{ }^{13} \mathrm{C}$ NMR $\left(101 \mathrm{MHz}, \mathrm{D}_{2} \mathrm{O}\right) \delta 174.01,44.31$, $37.99,29.77,28.22$. HRMS $m / z$ calcd for $\mathrm{C}_{7} \mathrm{H}_{13} \mathrm{O}_{2} \mathrm{~S}^{+}$is 161.0636 , found 161.0630 .

2-Methyl DMSP: obtained from DMS and 2-methylacrylic acid in a $15 \%$ yield as a white solid.

${ }^{1} \mathrm{H}$ NMR $\left(400 \mathrm{MHz}, \mathrm{CD}_{3} \mathrm{OD}\right) \delta 3.57(\mathrm{dd}, J=13.3,9.1 \mathrm{~Hz}, 1 \mathrm{H}), 3.44(\mathrm{dd}, J=13.3,5.1 \mathrm{~Hz}, 1 \mathrm{H})$, $3.15-3.08(\mathrm{~m}, 1 \mathrm{H}), 3.01$ (s, 3H), $3.00(\mathrm{~s}, 3 \mathrm{H}), 1.39$ (d, $J=7.2 \mathrm{~Hz}, 3 \mathrm{H}) ;{ }^{13} \mathrm{C}$ NMR $(101 \mathrm{MHz}$, $\left.\mathrm{CD}_{3} \mathrm{OD}\right) \delta 176.37,48.09,37.15,27.28,26.65,17.13$. HRMS $\mathrm{m} / z$ calcd for $\mathrm{C}_{6} \mathrm{H}_{13} \mathrm{O}_{2} \mathrm{~S}^{+}$is 149.0636, found 149.0640 .

3-Methyl DMSP: obtained from DMS and trans-3-methylacrylic acid in a $10 \%$ yield as a white solid.

${ }^{1} \mathrm{H}$ NMR (400 MHz, CD 30 O) $\delta 3.96-3.85(\mathrm{~m}, 1 \mathrm{H}), 3.01-2.91(\mathrm{~m}, 8 \mathrm{H}), 1.57(\mathrm{~d}, J=6.9 \mathrm{~Hz}$, $3 \mathrm{H}) ;{ }^{13} \mathrm{C}$ NMR $\left(101 \mathrm{MHz}, \mathrm{CD}_{3} \mathrm{OD}\right) \delta 172.65,48.82,36.87,23.99,22.35,14.63$. HRMS $\mathrm{m} / \mathrm{z}$ calcd for $\mathrm{C}_{6} \mathrm{H}_{13} \mathrm{O}_{2} \mathrm{~S}^{+}$is 149.0636 , found 149.0643 . 
Table 1: Kinetic parameters of the tested DMSP lyases

\begin{tabular}{|c|c|c|c|c|c|c|c|}
\hline Family & Species & $\begin{array}{c}\text { NCBI } \\
\text { Accession }\end{array}$ & $\begin{array}{c}\text { Specific } \\
\text { activity } \\
\left(\mu \mathrm{mol} \min ^{-1}\right. \\
\left.\text { mg enzyme }^{-1}\right)\end{array}$ & \begin{tabular}{|c|} 
Reported \\
Specific activity \\
$\left(\mu \mathrm{mol} \mathrm{min}^{-1} \mathrm{mg}\right.$ \\
enzyme $^{-1}$ )
\end{tabular} & $\begin{array}{l}k_{c a t} / K_{M} \\
\left(M^{-1} s^{-1}\right)\end{array}$ & $\begin{array}{c}K_{M} \\
(\mathrm{mM})\end{array}$ & $\begin{array}{l}k_{\text {cat }} \\
\left(s^{-1}\right)\end{array}$ \\
\hline Alma1 & Emiliania huxleyi & PODN21.1 & $381.6 \pm 69.7$ & $\sim 400$ (ref. $^{27}$ ) & $0.8^{*} 10^{5}$ & 9 & $7 * 10^{2}$ \\
\hline DddY & $\begin{array}{c}\text { Desulfovibrio } \\
\text { acrylicus }\end{array}$ & SHJ73420.1 & $1391 \pm 76$ & 2100 (ref. $^{25}$ ) & $1.32 * 10^{6}$ & 0.85 & $1.13 * 10^{3}$ \\
\hline \multirow[b]{2}{*}{ DddL } & Thioclava pacific & WP_051692700.1 & $\geq 83(1)$ & N.D. & 3181.7 & N.D. & N.D. \\
\hline & \begin{tabular}{|c|} 
Rhodobacter \\
sphaeroides 2.4 .1
\end{tabular} & Q3J6L0.1 & $\geq 70$ (1) & N.D. & 2683.3 & N.D. & N.D. \\
\hline DddQ & $\begin{array}{c}\text { Ruegeria } \\
\text { pomeroyi DSS-3 }\end{array}$ & AAV94883.1 & $2.22 \pm 0.04$ & $\begin{array}{l}2^{\sim} 5^{*} 10^{-3} \\
\text { (ref. } 16,23 \text { ) }\end{array}$ & 77.8 & N.D. & N.D. \\
\hline DddK & $\begin{array}{c}\text { Candidatus } \\
\text { Pelagibacter } \\
\text { ubique HTCC1062 }\end{array}$ & WP_011281678.1 & $15.24 \pm 0.53$ & 11.1 (ref. $^{30}$ ) & $419.1(2)$ & N.D. & N.D. (2) \\
\hline DddW & $\begin{array}{c}\text { Ruegeria } \\
\text { pomeroyi DSS-3 }\end{array}$ & WP_011046214.1 & $7.99 \pm 0.86$ & 61.2 (ref. $^{29}$ ) & $479(2)$ & N.D. (2) & N.D. (2) \\
\hline DddP & $\begin{array}{l}\text { Roseovarius } \\
\text { nubinhibens }\end{array}$ & WP_009813101.1 & $0.42 \pm 0.02$ & 0.31 (ref. $^{22}$ ) & N.D. & N.D. & N.D. \\
\hline
\end{tabular}

Footnotes:

N.D. not determined

${ }^{1}$ Specific activity was estimated from the activity and protein concentrations in the crude lysate; see accompanying paper.

${ }^{2}$ Initial rates showed linear dependency on substrate concentration throughout the tested range $\left([\mathrm{DMSP}]_{0} \leq 10 \mathrm{mM}\right)$. Hence, $k_{\text {cat }} / K_{M}$ could be deduced, but not the individual parameters (Supplemental Figure S1).

${ }^{3}$ The differences between the parameters reported here and in previous report may stem from different preparations, and also from different assay conditions (see text). 
Table 2: Cross-reactivity of the tested DMSP lyases with DMSP analogues. The activities with the various analogues are normalized to the activity of the same enzyme

\begin{tabular}{|c|c|c|c|c|c|c|c|}
\hline Enzyme & EMSP & DESP & CDESP & $\begin{array}{c}\text { 2-Methyl- } \\
\text { DMSP }\end{array}$ & $\begin{array}{c}\text { 3-Methyl- } \\
\text { DMSP }\end{array}$ & $I_{C R}$ & $*_{C R}$ \\
\hline Alma1 & $0 \pm 0.006$ & 0.00 & 0.00 & 0.00 & 0.00 & 0.00 & 0.00 \\
\hline DddY & $0.42 \pm 0.04$ & $0.0040 \pm 0.002$ & $0.07 \pm 0.003$ & 0.00 & $0.03 \pm 0.004$ & 0.10 & 0.51 \\
\hline DddL & $0.01 \pm 0.004$ & 0.00 & 0.00 & 0.00 & 0.00 & 0.0017 & 0.008 \\
\hline DddQ & $0.59 \pm 0.009$ & $0.91 \pm 0.14$ & $0.70 \pm 0.11$ & 0.00 & $18.78 \pm 0.02$ & 4.19 & 4.71 \\
\hline DddW & $0.39 \pm 0.04$ & $0.55 \pm 0.04$ & $0.94 \pm 0.04$ & $0.09 \pm 0.01$ & 0.00 & 0.39 & 2.31 \\
\hline DddK & $0.69 \pm 0.04$ & $0.61 \pm 0.005$ & $0.55 \pm 0.02$ & $0.02 \pm 0.001$ & $0.02 \pm 0.003$ & 0.38 & 2.04 \\
\hline DddP & $0.32 \pm 0.04$ & $0.89 \pm 0.03$ & 0.00 & $0.88 \pm 0.09$ & $0.66 \pm 0.08$ & 0.55 & 3.45 \\
\hline STDEV & 0.25 & 0.39 & 0.37 & 0.30 & 6.53 & & \\
\hline $\begin{array}{c}\text { Mean } \\
\text { Coefficient } \\
\text { of variation } \\
\text { (C }{ }_{\mathrm{v}}{ }^{\text {) }}\end{array}$ & 0.35 & 0.42 & 0.32 & 0.14 & 2.79 & & \\
\hline
\end{tabular}

with DMSP. 


\section{References}

1. Khersonsky, O., and Tawfik, D. S. (2010) Enzyme promiscuity: a mechanistic and evolutionary perspective, Annual review of biochemistry 79, 471-505.

2. Pandya, C., Farelli, J. D., Dunaway-Mariano, D., and Allen, K. N. (2014) Enzyme promiscuity: engine of evolutionary innovation, The Journal of biological chemistry 289, 30229-30236.

3. Miao, Y., Rahimi, M., Geertsema, E. M., and Poelarends, G. J. (2015) Recent developments in enzyme promiscuity for carbon-carbon bond-forming reactions, Current opinion in chemical biology 25, 115-123.

4. Boyd, E. S., and Peters, J. W. (2013) New insights into the evolutionary history of biological nitrogen fixation, Frontiers in microbiology 4, 201.

5. Dobbek, H. (2017) Nitrogenase: A fuel-producing microbe, Nature chemical biology 13, 134-135.

6. Hardy, R. W. F., Burns, R. C., and Holsten, R. D. (1973) Applications of the acetyleneethylene assay for measurement of nitrogen fixation, Soil Biology and Biochemistry 5 , 47-81.

7. Van Loo, B., and Hollfelder, F. (2010) Enzyme Promiscuity and Evolution of New Protein Functions, In Manual of Industrial Microbiology and Biotechnology, Third Edition, American Society of Microbiology.

8. Gerlt, J. A., Babbitt, P. C., Jacobson, M. P., and Almo, S. C. (2012) Divergent evolution in enolase superfamily: strategies for assigning functions, The Journal of biological chemistry 287, 29-34.

9. Roodveldt, C., and Tawfik, D. S. (2005) Shared promiscuous activities and evolutionary features in various members of the amidohydrolase superfamily, Biochemistry 44, 12728-12736.

10. Schnoes, A. M., Brown, S. D., Dodevski, I., and Babbitt, P. C. (2009) Annotation error in public databases: misannotation of molecular function in enzyme superfamilies, PLoS computational biology 5, e1000605.

11. Khersonsky, O., and Tawfik, D. S. (2005) Structure-reactivity studies of serum paraoxonase PON1 suggest that its native activity is lactonase, Biochemistry 44, 63716382.

12. Bar-Even, A., Noor, E., Savir, Y., Liebermeister, W., Davidi, D., Tawfik, D. S., and Milo, R. (2011) The moderately efficient enzyme: evolutionary and physicochemical trends shaping enzyme parameters, Biochemistry 50, 4402-4410.

13. Todd, J. D., Rogers, R., Li, Y. G., Wexler, M., Bond, P. L., Sun, L., Curson, A. R., Malin, G., Steinke, M., and Johnston, A. W. (2007) Structural and regulatory genes required to make the gas dimethyl sulfide in bacteria, Science 315, 666-669.

14. Alcolombri, U., Laurino, P., Lara-Astiaso, P., Vardi, A., and Tawfik, D. S. (2014) DddD is a CoA-transferase/lyase producing dimethyl sulfide in the marine environment, Biochemistry 53, 5473-5475.

15. Todd, J. D., Curson, A. R., Dupont, C. L., Nicholson, P., and Johnston, A. W. (2009) The dddP gene, encoding a novel enzyme that converts dimethylsulfoniopropionate into dimethyl sulfide, is widespread in ocean metagenomes and marine bacteria and also occurs in some Ascomycete fungi, Environmental microbiology 11, 1376-1385. 
16. Todd, J. D., Curson, A. R., Kirkwood, M., Sullivan, M. J., Green, R. T., and Johnston, A. W. (2011) DddQ, a novel, cupin-containing, dimethylsulfoniopropionate lyase in marine roseobacters and in uncultured marine bacteria, Environmental microbiology 13, 427438.

17. Curson, A. R., Rogers, R., Todd, J. D., Brearley, C. A., and Johnston, A. W. (2008) Molecular genetic analysis of a dimethylsulfoniopropionate lyase that liberates the climate-changing gas dimethylsulfide in several marine alpha-proteobacteria and Rhodobacter sphaeroides, Environmental microbiology 10, 757-767.

18. Todd, J. D., Kirkwood, M., Newton-Payne, S., and Johnston, A. W. (2012) DddW, a third DMSP lyase in a model Roseobacter marine bacterium, Ruegeria pomeroyi DSS-3, The ISME journal 6, 223-226.

19. Sun, J., Todd, J. D., Thrash, J. C., Qian, Y., Qian, M. C., Temperton, B., Guo, J., Fowler, E. K., Aldrich, J. T., Nicora, C. D., Lipton, M. S., Smith, R. D., De Leenheer, P., Payne, S. H., Johnston, A. W., Davie-Martin, C. L., Halsey, K. H., and Giovannoni, S. J. (2016) The abundant marine bacterium Pelagibacter simultaneously catabolizes dimethylsulfoniopropionate to the gases dimethyl sulfide and methanethiol, Nature microbiology 1, 16065.

20. de Souza, M. P., and Yoch, D. C. (1995) Purification and characterization of dimethylsulfoniopropionate lyase from an alcaligenes-like dimethyl sulfide-producing marine isolate, Applied and environmental microbiology 61, 21-26.

21. Curson, A. R., Sullivan, M. J., Todd, J. D., and Johnston, A. W. (2011) DddY, a periplasmic dimethylsulfoniopropionate lyase found in taxonomically diverse species of Proteobacteria, The ISME journal 5, 1191-1200.

22. Kirkwood, M., Le Brun, N. E., Todd, J. D., and Johnston, A. W. (2010) The dddP gene of Roseovarius nubinhibens encodes a novel lyase that cleaves dimethylsulfoniopropionate into acrylate plus dimethyl sulfide, Microbiology 156, 1900-1906.

23. Alcolombri, U., Elias, M., Vardi, A., and Tawfik, D. S. (2014) Ambiguous evidence for assigning DddQ as a dimethylsulfoniopropionate lyase and oceanic dimethylsulfide producer, Proceedings of the National Academy of Sciences of the United States of America 111, E2078-2079.

24. Li, C. Y., Wei, T. D., Zhang, S. H., Chen, X. L., Gao, X., Wang, P., Xie, B. B., Su, H. N., Qin, Q. L., Zhang, X. Y., Yu, J., Zhang, H. H., Zhou, B. C., Yang, G. P., and Zhang, Y. Z. (2014) Molecular insight into bacterial cleavage of oceanic dimethylsulfoniopropionate into dimethyl sulfide, Proceedings of the National Academy of Sciences of the United States of America 111, 1026-1031.

25. van der Maarel, M. J. E. C., Aukema, W., and Hansen, T. A. (1996) Purification and characterization of a dimethylsulfoniopropionate cleaving enzyme from Desulfovibrio acrylicus, FEMS Microbiology Letters 143, 241-245.

26. James, L. C., and Tawfik, D. S. (2003) The specificity of cross-reactivity: promiscuous antibody binding involves specific hydrogen bonds rather than nonspecific hydrophobic stickiness, Protein science : a publication of the Protein Society 12, 2183-2193.

27. Alcolombri, U., Ben-Dor, S., Feldmesser, E., Levin, Y., Tawfik, D. S., and Vardi, A. (2015) MARINE SULFUR CYCLE. Identification of the algal dimethyl sulfide-releasing enzyme: A missing link in the marine sulfur cycle, Science 348, 1466-1469.

28. Brummett, A. E., and Dey, M. (2016) New Mechanistic Insight from Substrate- and Product-Bound Structures of the Metal-Dependent Dimethylsulfoniopropionate Lyase DddQ, Biochemistry 55, 6162-6174. 
29. Brummett, A. E., Schnicker, N. J., Crider, A., Todd, J. D., and Dey, M. (2015) Biochemical, Kinetic, and Spectroscopic Characterization of Ruegeria pomeroyi DddW--A Mononuclear Iron-Dependent DMSP Lyase, PloS one 10, e0127288.

30. Schnicker, N. J., De Silva, S. M., Todd, J. D., and Dey, M. (2017) Structural and Biochemical Insights into Dimethylsulfoniopropionate Cleavage by Cofactor-Bound DddK from the Prolific Marine Bacterium Pelagibacter, Biochemistry 56, 2873-2885.

31. Baier, F., Chen, J., Solomonson, M., Strynadka, N. C., and Tokuriki, N. (2015) Distinct Metal Isoforms Underlie Promiscuous Activity Profiles of Metalloenzymes, ACS Chem Biol 10, 1684-1693.

32. Nath, A., and Atkins, W. M. (2008) A quantitative index of substrate promiscuity, Biochemistry 47, 157-166.

33. Tohge, T., Watanabe, M., Hoefgen, R., and Fernie, A. R. (2013) Shikimate and phenylalanine biosynthesis in the green lineage, Frontiers in plant science 4, 62 .

34. Seelig, B. (2017) Multifunctional enzymes from reduced genomes - model proteins for simple primordial metabolism?, Molecular microbiology.

35. Menefee, A. L., and Zeczycki, T. N. (2014) Nearly 50 years in the making: defining the catalytic mechanism of the multifunctional enzyme, pyruvate carboxylase, The FEBS journal 281, 1333-1354.

36. Lorenzen, W., Ahrendt, T., Bozhuyuk, K. A., and Bode, H. B. (2014) A multifunctional enzyme is involved in bacterial ether lipid biosynthesis, Nature chemical biology 10, 425427.

37. Alcolombri, U., Lei, L., Meltzer, D., Vardi, A., and Tawfik, D. S. (2016) Assigning the algal source of dimethylsulfide using a selective lyase inhibitor, ACS Chemical Biology. 


\section{Figure Legends}

Figure 1. Profiling of DMSP lyases with a series of DMSP analogues. (A) The applied DMSP analogue. The dialkylsulfide leaving groups are denoted in red, and bold lines represent the modifications relative to DMSP. (B) The relative rates of elimination compared to the activity of these enzymes with DMSP. All substrates were applied at 10mM. Enzymes tested: Alma1 (Emiliana huxleyi Alma1, ${ }^{27} 0.3 \mu \mathrm{g} / \mathrm{mL}$, DMS releasing rate, $114.5 \mu \mathrm{M} / \mathrm{min}$ ); DddY (DaDddY, described in accompany paper, $20 \mathrm{ng} / \mathrm{mL}$, DMS releasing rate, $27.8 \mathrm{\mu M} / \mathrm{min}) ; \mathrm{DddL}^{17}$ (RsDddL; crude lysate, estimated enzyme concentration of $8 \mu \mathrm{g} / \mathrm{mL}$ (see accompanying paper), DMS releasing rate, 615.9 $\mu \mathrm{M} / \mathrm{min}$ ); $\mathrm{DddQ}^{16}$ (Ruegeria pomeroyi DSS-3, $100 \mu \mathrm{g} / \mathrm{mL}$, DMS releasing rate, 13.2 $\mu \mathrm{M} / \mathrm{min}$ ); $\mathrm{DddW}^{18}$ (Ruegeria pomeroyi DSS-3, $15 \mu \mathrm{g} / \mathrm{mL}$, DMS releasing rate, 119.9 $\mu \mathrm{M} / \mathrm{min}$ ); $\operatorname{DddK}^{19}$ (Candidatus Pelagibacter ubique HTCC1062, $8 \mu \mathrm{g} / \mathrm{mL}$, DMS releasing rate, $121.92 \mu \mathrm{M} / \mathrm{min}$ ); DddP ${ }^{22}$ (Roseovarius nubinhibens, $10 \mathrm{ug} / \mathrm{mL}$, DMS releasing rate, $4.24 \mu \mathrm{M} / \mathrm{min})$.

Figure 2. The DMSP lyase activity of the tested enzymes correlates with their selectivity toward DMSP. The Y-axis denotes the specific activity of the 7 enzymes tested (specified in Table 1) normalized by the enzyme's molecular weight (the units are $\mu$ mol DMS per min per $\mu \mathrm{mol}$ enzyme) and presented on a log2 scale. The $x$-axis denotes these enzymes' cross-reactivity by two indexes: (A) $I_{C R}$ - the average relative activity with the 5 DMSP analogues (Equation 1); (B) ${ }^{*} I_{C R}-$ a parameterized index of relative activities (Equation 2). 

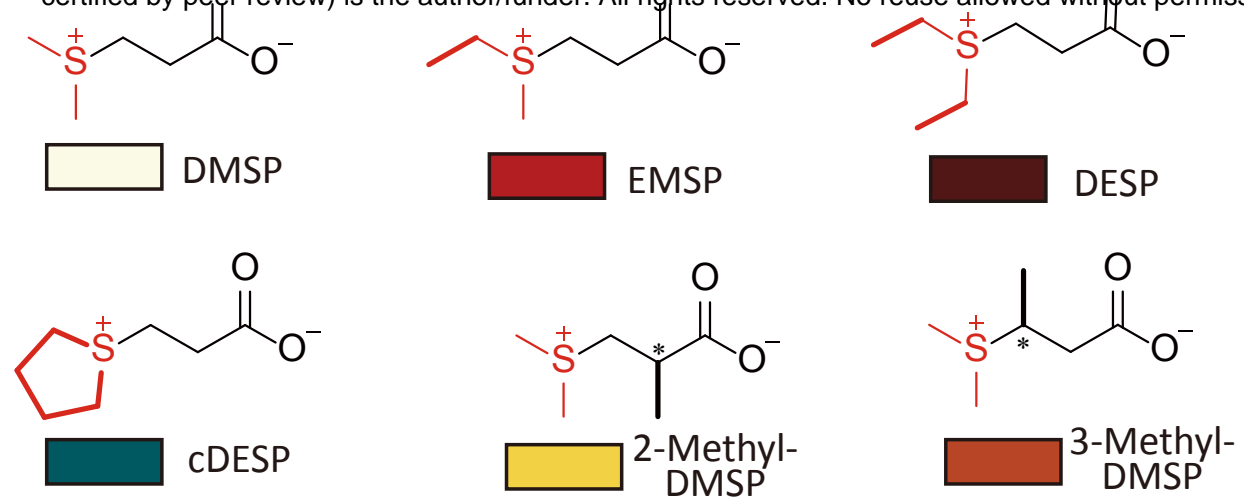

B

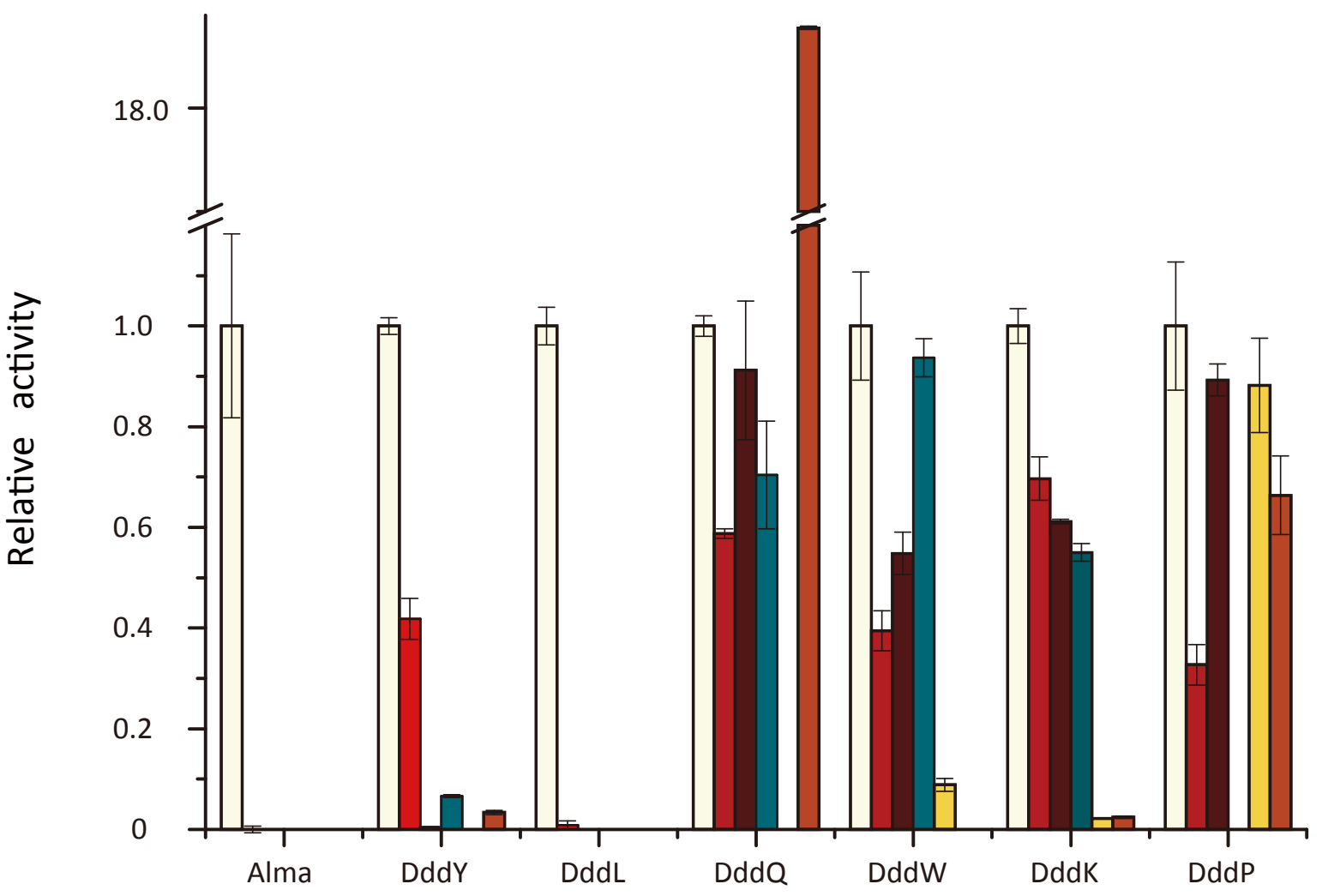

Figure 1. Profiling of DMSP lyases with a series of DMSP analogues. (A) The applied DMSP analogue. The dialkylsulfide leaving groups are denoted in red, and bold lines represent the modifications relative to DMSP. (B) The relative rates of elimination compared to the activity of these enzymes with DMSP. All substrates were applied at $10 \mathrm{mM}$. Enzymes tested: Alma1 (Emiliana huxleyi Alma1, ${ }^{27} 0.3$ $\mu \mathrm{g} / \mathrm{mL}$, DMS releasing rate, $114.5 \mu \mathrm{M} / \mathrm{min}$ ); DddY (DaDddY, described in accompany paper, 20 $\mathrm{ng} / \mathrm{mL}$, DMS releasing rate, $27.8 \mu \mathrm{M} / \mathrm{min}$ ); DddL ${ }^{17}$ (RsDddL; crude lysate, estimated enzyme concentration of $8 \mu \mathrm{g} / \mathrm{mL}$ (see accompanying paper), DMS releasing rate, $615.9 \mu \mathrm{M} / \mathrm{min}$ ); DddQ ${ }^{16}$ (Ruegeria pomeroyi DSS-3, $100 \mu \mathrm{g} / \mathrm{mL}$, DMS releasing rate, $13.2 \mu \mathrm{M} / \mathrm{min}$ ); DddW ${ }^{18}$ (Ruegeria pomeroyi DSS-3, $15 \mu \mathrm{g} / \mathrm{mL}$, DMS releasing rate, $119.9 \mu \mathrm{M} / \mathrm{min}$ ); DddK ${ }^{19}$ (Candidatus Pelagibacter ubique HTCC1062, $8 \mu \mathrm{g} / \mathrm{mL}$, DMS releasing rate, $121.92 \mu \mathrm{M} / \mathrm{min}$ ); DddP 22 (Roseovarius nubinhibens, 10ug/mL, DMS releasing rate, $4.24 \mu \mathrm{M} / \mathrm{min})$. 


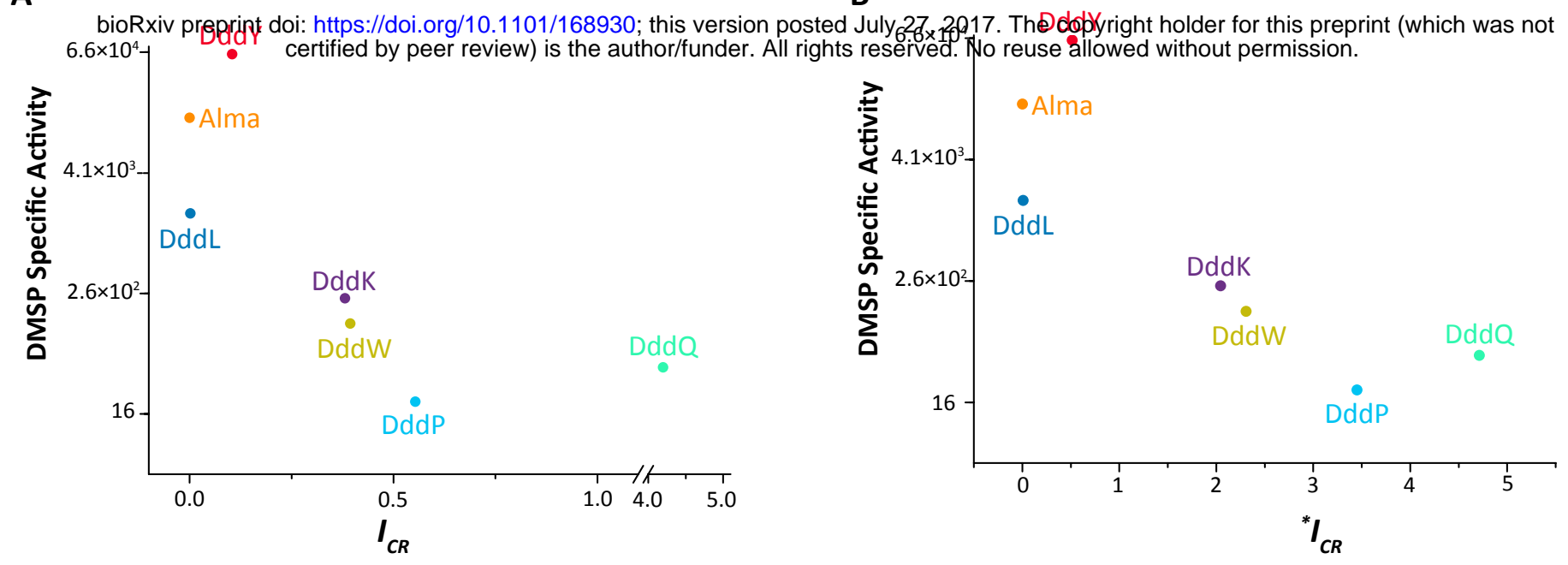

B

Figure 2. The DMSP lyase activity of the tested enzymes correlates with their selectivity toward DMSP. The Y-axis denotes the specific activity of the 7 enzymes tested (specified in Table 1) normalized by the enzyme's molecular weight (the units are $\mu \mathrm{mol} D M S$ per min per $\mu \mathrm{mol}$ enzyme) and presented on a log2 scale. The $x$-axis denotes these enzymes' cross-reactivity by two indexes: $(A) I_{C R}$ - the average relative activity with the 5 DMSP analogues (Equation 1); (B) ${ }^{*} I_{C R}$ - a parameterized index of relative activities (Equation 2). 